\title{
Dust evolution in photoevaporating protoplanetary disks
}

\author{
H. Nomura ${ }^{1}$, Y. Aikawa ${ }^{1}$, S. Inutsuka ${ }^{2}$ and Y. Nakagawa ${ }^{1}$ \\ ${ }^{1}$ Dept. of Earth and Planetary Sciences, Kobe University, Kobe 657-8501, Japan \\ ${ }^{2}$ Dept. of Physics, Graduate School of Science, Kyoto University, Kyoto 606-8502, Japan
}

\begin{abstract}
We construct a model of the physical structure of photoevaporating protoplanetary disks, and numerically calculate the coagulation and settling/evaporating process of dust particles in the disks. Our result show that (sub)micron-sized-dust-particles could evaporate with the gas, which leads to dispersal of infrared excess radiation from the disks.
\end{abstract}

Photoevaporation of protoplanetary disks induced by ultraviolet photons and/or X-rays from the central stars is known as one of possible mechanisms of gaseous disk dispersal. In addition, photoevaporating flow is expected to affect dust evolution that will lead to the planet formation in the disks, and observational properties of the disks.

In this work we have made a detailed model of gas density, temperature, and velocity structure of one-dimensional, steady photoevaporating flow in protoplanetary disks. By using the obtained disk structure, we compare the gravitational and the gas friction forces which affect on a dust particle in the disks to estimate a critical dust radius. The result shows that (sub)micronsized-dust-particles could evaporate with the gas, instead of settling toward the disk midplane (Figure 1, left; Nomura \& Inutsuka 2004). In addition, we calculate the dust evolution in the disks by numerically solving a coagulation equation for the dust particles (Nomura \& Nakagawa 2006), taking into account the upward motion of the dust grains, which shows that (sub)micronsized-dust-particles disappear from the disks because they can move only upward with the flow as well as coagulate very quickly near the dense disk midplane. Finally, making use of the resulting spatial and size distributions of the dust particles, we calculate spectral energy distributions (SEDs) of dust continuum emission from the disks, which suggests that the photoevaporation process could help to reduce infrared excess radiation from the disks (Figure 1, right).
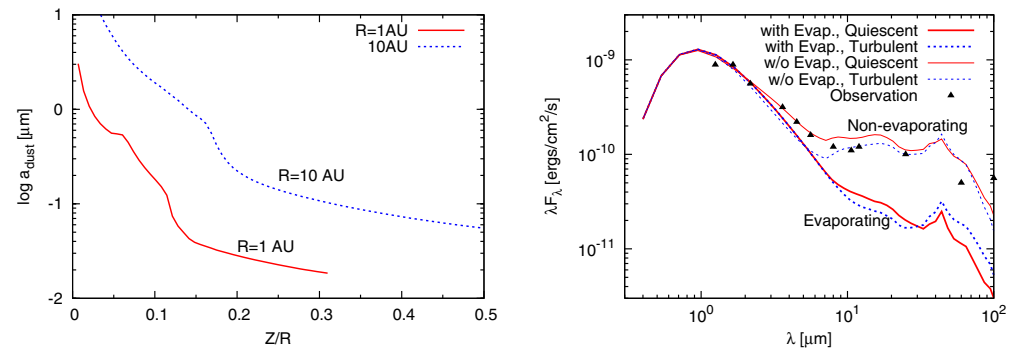

Figure 1. The critical radius of a dust particle at various positions $(R, Z)$ in the disk (left). The resulting SEDs of evaporating (thick lines) and non-evaporating (thin lines) disks with observational median SED toward classical T Tauri stars (triangles; D'Alessio et al. 2006) (right).

\section{References}

D'Alessio, P., Calvet, N., Hartmann, L., Franco-Hernandez, R., \& Servin, H. 2006, ApJ 638, 314 Nomura, H. \& Inutsuka, S. 2004, in: J.-P. Beaulieu, A. Lecavelier Des Etangs \& C. Terquem (eds.), Extrasolar Planets: Today and Tomorrow (ASP-CS) 321, 335

Nomura, H. \& Nakagawa, Y. 2006, ApJ 640, 1099 